\title{
L'ecotomografia nelle urgenze nefrologiche
}

\author{
B. Ravasi ${ }^{1}$, E. Gilardoni ${ }^{2}$, G. Buccianti ${ }^{1}$ \\ ${ }^{1}$ Servizio di Nefrologia e Dialisi, Ospedale Maggiore IRCCS, Milano \\ ${ }^{2}$ Servizio di Ecotomografia, Presidio Ospedaliero Uboldo, USSL 58, \\ Cernusco Sul Naviglio
}

$\mathrm{n}$ assenza di strumenti diagnostici invasivi e non invasivi, la diagnosi e la valutazione dell'urgenza medica erano affidate esclusivamente alle capacità del medico, al suo intuito ed al suo senso critico. L'introduzione di tecniche diagnostiche con o senza l'utilizzo di mezzo di contrasto, se da un lato ha consentito una diagnosi più accurata $e$ certa, dall'altro ha comportato un allungamento dei tempi tecnici di attesa che il carattere di urgenza di alcuni quadri clinici spesso non consente.

L'ecotomografia restituisce al medico la capacità diagnostica consentendogli in tempo reale di effettuare una diagnosi accurata e di programmare un intervento terapeutico. Tuttavia, l'assenza di una efficace caratterizzazione tissutale mediante ultrasuoni non consente ancora una diagnosi specifica di talune condizioni morbose che richiederanno l'impiego di indagini sofisticate non più in senso diagnostico assoluto ma solo a conferma di una diagnosi presunta (1).

Nella pratica clinica nefrologica possono manifestarsi patologie che rivestono carattere di urgenza, nelle quali una diagnosi precoce costituisce premessa essenziale per una completa restitutio ad integrum dell'organo interessato.

Nel presente lavoro verranno valutati i quadri ecografici e clinici delle principali condizioni di urgenza nefrologica nel bambino, nell'adulto e nell'anziano.

\section{Metodologia}

Questo studio è stato condotto dal nostro Servizio di Ecotomografia (Servizio di Nefrologia e Dialisi, Ospedale Maggiore di Milano) in collaborazione con il Servizio di Ecotomografia del Presidio Ospedaliero "Uboldo" di Cernusco Sul Naviglio, USSL 58.

Gli ecotomografi da noi utilizzati sono stati i seguenti: modello RT-x 400 della General Electric dotato di sonda convex 3.5 Mhz e lineare 7.5 Mhz; e modello ATL Ultramark 5 dotato di sonde settoriali 3.5 e $5 \mathrm{Mhz}$, e lineare $7.5 \mathrm{Mhz}$.

I quadri clinici da noi esaminati, compatibili con una situazione di urgenza nefrologica, presentavano una sintomatologia algica addomino-lombare aspecficia o un episodio accessionale di colica franca, macroematuria e/o oligo-anuria, in associazione o con la prevalenza di almeno uno di tali sintomi, ed in considerazione di tali condizioni cliniche dei pazienti non è stato possibile usufruire di una preparazione addominale per l'esecuzione dell'indagine ecotomografica. Nei casi in cui era possibile si richiese il riempimento della vescica da utilizzare quale finestra acustica pelvica.

Ci siamo avvalsi di scansioni ecografiche standard nell'età adulta ed avanzata, adattando la frequenza dei trasduttori utilizzati al tipo somatico del paziente, e nell'età pediatrica abbiamo integrato le comuni proiezioni con un approccio posteriore per lo studio delle logge renali in relazione al meteorismo intestinale, solitamente più intenso nell'infanzia (2), prediligendo comunque i trasduttori con una frequenza maggiore ( 5 e $7.5 \mathrm{Mhz}$ ).

\section{L'ecotomografia nelle urgenze nefrologiche pediatriche}

Le urgenze nefrologiche nell'età pediatrica riconoscono come cause più frequenti le anomalie congenite renali, la patologia ostruttiva e quella neoplastica. Le anomalie renali congenite rappresentano più spesso il risultato di un difetto di sviluppo durante il periodo fetale rispetto ad una patologia trasmessa geneticamente, e la nostra attenzione si rivolgerà essenzialmente alla sindrome del giunto pielo-ureterale ed al reflusso vescico ureterale.

La sindrome del giunto pielo-ureterale è la causa più frequente di uropatia ostruttiva in età pediatrica, essenzialmente di tipo organico con rare forme funzionali. Sindromi dell'uretere sottogiuntale e della giunzione uretero-vescicale sono più rare.

Le cause possono essere alterazioni intrinseche congenite quali ipertrofia della muscolare, valvole, inserzione anomala 
del giunto, o alterazioni intrinseche acquisite quali calcoli, miceti o neoplasie, oppure alterazioni ab extrinseco come vasi iliaci anomali o l'uretere retrocavale. La stenosi può essere più o meno serrata, mono o bilaterale, non di rado si può rilevare associazione con altre malformazioni delle vie escretrici (3).

La sintomatologia può essere del tutto aspecifica, presentando il bimbo febbre e malessere generale (in considerazione del fatto che i sintomi urologici sono presenti a partire dalla II infanzia in poi), infezioni delle vie urinarie acute o recidivanti, dolore in regione lombare, e se l'affezione è bilaterale insufficienza renale.

L'iconografia ecotomografica è la seguente: dilatazione più $\mathrm{o}$ meno marcata dell'ampolla renale (di aspetto rotondeggiante ovalare) associata ad una diffusa ectasia dei calici. Non esiste di norma una dilatazione dell'uretere sottogiuntale. Si rileva inoltre uno spessore parenchimale ridotto correlato al grado della idronefrosi, a sua volta dipendente dalla causa stenotica, da confrontare con il rene controlaterale.

Utile eventualmente uno studio a paziente prono per una migliore esplorabilità, ovviando al problema dell'itenso meteorismo intestinale del bambino, e per una verifica di modificazioni eventuali del volume dell'ectasia ampollare, espressione questa di una idronefrosi intermittente suggestiva per una forma funzionale o di compressione ab extrinseco (2).

Nelle forme intermedie può essere utile uno studio basale e dopo carico idrico, tale "ecografia diuretica" è causa talvolta di crisi dolorose tipo colica dovute alla rapida distensione del bacinetto renale nelle fasi di diuresi abbondante (3).

Per reflusso vescico-ureterale primario si intende la risalita di urina in uretere/i durante la minzione, affezione relativamente frequente nel bambino e causa importante di danno pielonefritico (nefrite scleroatrofica interstiziale da reflusso).

La causa è generalmente rappresentata da una alterazione congenita con imperfetta funzione della valvola vescico-ureterale, più frequentemente per eccessiva brevità del tratto intramurale degli ureteri, o per difettoso funzinamento dell'apparato valvolare di contenzione che assicura la chiusura del tratto intramurale dell'uretere in caso di minzione, anomalia talora promossa da fenomeni flogistici cisto-ureterali: Alcune forme di reflusso invece, possono essere secondarie ad ostruzione sottovescicale o a turbe del funzionamento della minzione.

Il $30-50 \%$ dei bambini con infezioni recidivanti delle vie urinarie è interessato, più spesso nel primo anno di vita (4), e tale affezione è responsabile del $30 \%$ circa dei casi di IRC al di sotto dei 20 anni (5).

I sintomi possono essere aspecifici come poliuria, anemia ed ipertensione, oppure il bimbo può presentare una infezione acuta delle vie urinarie o epidosi recidivanti con il quadro clinico della pielonefrite cronica.

L'ecotomografia non è l'indagine diagnostica più sensibile nel rilevare la presenza di un reflusso di I o II grado, ancora reversibile e di modesta entità. In questi casi l'unico segno ecografico è aspecifico trattandosi di una modica dilatazione della porzione terminale dell'uretere. Tale metodica risulta invece utile nell'iter diagnostico delle fasi avanzate di reflussi di III o IV grado con marcata dilatazione delle vie escretici, e nel follow-up delle stesse consentendo una valutazione degli effetti della stasi sul parenchima renale (2). Il parenchima renale leso risulta iperecogneo, assottigliato, con diminuita distanza tra il profilo renale ed il fondo dei calici solitamente ben individuabili, ampi ed arrotondati. Talora si rilevano incisure più o meno grossolane del profilo renale distinguibili dalle fisiologiche introflessioni interlobari (3).

Completano l'iter diagnostico test urodinamici, quali la cistografia minzionale, la cistomanometria e la uroflussimetria.

Le urgenze nefrologiche in età pediatrica possono avere quale causa anche una $\mathrm{pa}$ tologia dismetabolica ossia errori congeniti del metabolismo come per esempio ossalosi, cistinuria, ipercalcemia idiopatica e litiasi calcica.

Ossalosi può essere dovuta ad alterazioni metaboliche congenite da deficit enzimatici che determinano iperossaluria (ossalosi primaria) o eccessiva introduzione con la dieta (cioccolato) o eccessivo assorbimento enterico (iperossaluria enterica) oppure eccessiva produzione (ossalosi secondaria). L'ossalosi è causa di una nefrite interstiziale cronica per depressione di ossalato di calcio nei tubuli e nell'interstizio ed il quadro ecografico è espresso da una nefrocalcinosi corticomidollare $(6,7)$.

Cistinuria è la più frequente forma di aminoaciduria, malattia ereditaria a tra- smissione autosomica recessiva, con espressione variabile negli eterozigoti, caratterizzata dall'aumento della escrezione di aminoacidi dibasici (lisina, ornitina, arginina e cistina) per difetto tubulare. Solo la eccessiva escrezione di cistina provoca la cristallizzazione e la formazione di calcoli, per la scarsa solubilità di questo aminoacido. Da non confondere con la cistinosi, la causa più frequente di sindrome di Fanconi, malattia ereditaria a trasmissione autosomica recessiva con deposizione intracellulare di cistina in vari organi, tra i quali il rene. Ipercalcemia idiopatica determina deposizione di sali di calcio prima nelle strutture midollari (tubuli collettori ed anse di Henle) e successivamente nel parenchima renale con cosneguente quadro ecotomografico caratteristico per la nefrocalcinosi con piramidi iperecogene e con progressivo interessamento tubulare e quindi iperecogenicità dalla periferia delle piramidi verso lo sbocco dei dotti collettori.

La sintomatologia di queste affezioni è comune ai diversi tipi di nefrolitiasi indipendentemente dalla natura litiasica. Il dolore è il sintomo più frequente, di tipo accessionale, in sede lombare irradiato in basso ed anteriormente verso la regione sovrapubica ed i genitali esterni.

Il paziente si mostra agitato, talora sono presenti nausea e/o vomito, stranguria, pollachiuria, ematuria macroscopica ed infezione urinaria con il quadro clinico della pielonefrite acuta.

Un altro importante capitolo delle urgenze in nefrologia è rappresentato dalla patologia neoplastica benigna o maligna, in cui frequentemente il primo ed unico segno è una massa palpabile a livello addominale.

Il nefroblastoma o tumore di Wilms è la neoplasia renale più frequente in età pediatrica, rappresenta il $6.2 \%$ di tutti i tumori solidi infantili, ha una prevalenza di 0.78/100.000 bambini con età inferiore ai 14 anni, colpisce più frequentemente bambini da 1 a 6 anni con massima incidenza intorno ai 3 anni, e nel 5-10\% dei casi risulta essere bilaterale $(2,4,8)$.

All'esame obbiettivo si apprezza una voluminosa massa in sede ipocondriaca o al fianco, talora con diametro superiore ai $10 \mathrm{~cm}$, visibile e/o palpabile, non mobile con gli atti respiratori, con tendenza a dislocare piuttosto che ad infiltrare gli organi contigui. Nel 20-30\% dei casi l'unico segno della neoplasia è uno scadi- 


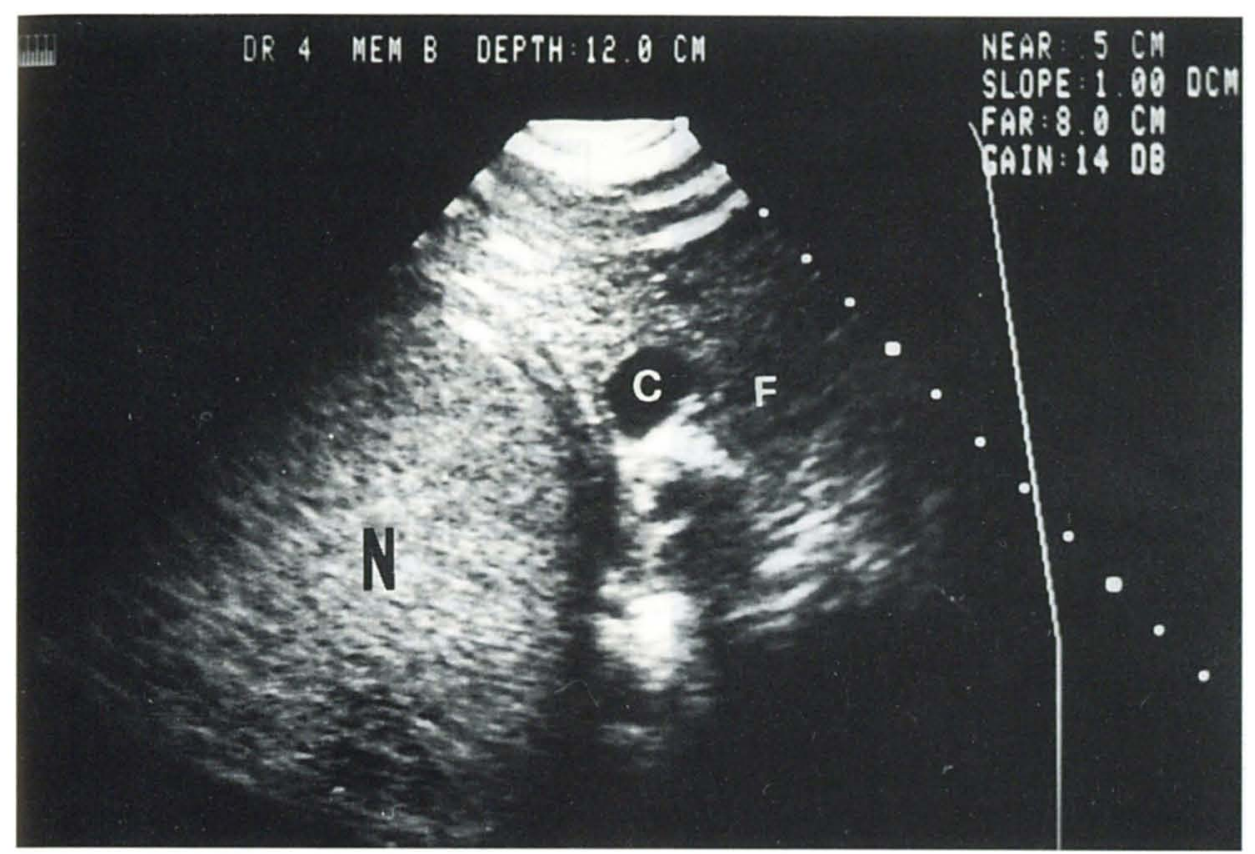

Fig. 1 - Scansione trasversale anteriore dell' ipocondrio destro eseguita con paziente in decubito supino. In bambino di 18 mesi, si rileva voluminosa neoformazione eteroproduttiva $(N)$ ad ecostruttura grossolana e disomogenea, con totale sostituzione del parenchima renale destro, non visualizzabile. $C=C$ Colecisti; $F=$ Fegato.

mento delle condizioni generali con sintomi aspecifici quali astenia ed anoressia, oppure sintomi clinici quale dolore addominale non caratteristico ed ematuria macroscopica $(5,8)$.

All'esame ecotomografico si evidenzia una massa solida parenchimatosa di dimensioni variabili ad ecostruttura isoecogena con il restante parenchima ed omogenea, soprattutto nelle fasi iniziali, talora iperecognea, e con l'aumentare delle dimensioni aumenta la disomogeneità ecostrutturale per sviluppo di aree ipo-anecogene dovute a necrosi intraneoplastiche (3) (Fig. 1). Di norma tale neoplasia non contiene clacificazioni e non si accompagna a linfoadenomegalie loco-regionali a differenza del neuroblastoma (2).

Valutare sempre 1'eventuale interessamento della vena renale omolaterale e della vena cava, del rene controlaterale, di eventuali metastasi linfoghiandolari retroperitoneali o metastasi epatiche.

Tra le patologie neoplastiche maligne possiamo citare il linfoma sistemico o leucemia che si manifesta con due possibili quadri di infiltrazione parenchimale, diffusa o focale.

Segni ecotomografici sono un aumento delle dimensioni del rene, una diminuzione della ecogenicità del parenchima, una

\section{L'ecotomografia nelle urgenze nefrologiche dell'età adulta}

L'eziopatogenesi delle urgenze nefrologiche nell'età adulta riconosce più frequentemente come substrato la patologia malformativa o litiasica, eventuali complicanze a carico delle lesioni cistiche oppure una patologia tumorale.

L'agenesia renale monolaterale o assenza congenita di un rene, affezione più frequente a sinistra e nel maschio, rappresenta di per sé una condizione asintomatica costituendo a volte un reperto del tutto occasionale in corso di indagine ecotomografica o urografica, ma assumendo clinicamente una importanza rilevante in caso di insulto traumatico o clinico dell'unico rene funzionante e caratteristicamente ipertrofico per compenso. La sintomatologia varia dall'accesso colico in rene unico alla insufficienza renale acuta o cronica. L'indagine ecografica rivela una loggia renale libera dalla propria immagine renale ed indirizza l'iter diagnostico per una successiva conferma. Un'altra affezione malformativa è la sindrome del giunto pielo-ureterale (Fig. 2) che può divenire manifesta in età adulta per complicanze settiche simulanti un quadro di addome acuto, o per un
Tra le neoplasie benigne la cisti multiloculare presenta obbiettività e clinica simile al nefroblastoma ma quadro ecotomografico caratteristico per immagine anecogena di notevoli dimensioni e settata (3).

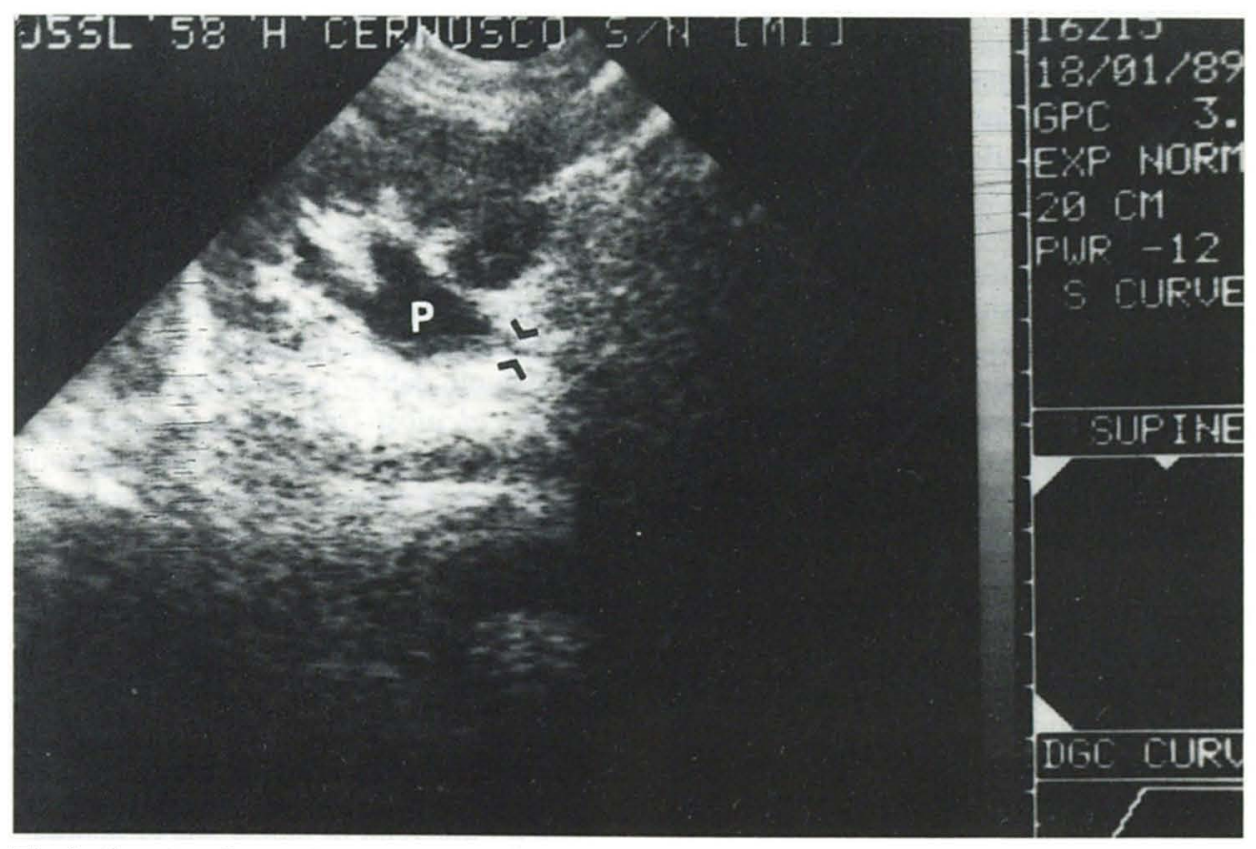

Fig. 2 - Scansione longitudinale obliqua posteriore dell' ipocondrio sinistro eseguita con paziente in ortostatismo. Si rileva dilatazione delle vie escretrici che si configura nella modesta pielectasia $(P)$ e nella visualizzazione del tratto iniziale filiforme dell' uretere $(><)$ "Sindrome del colletto". 


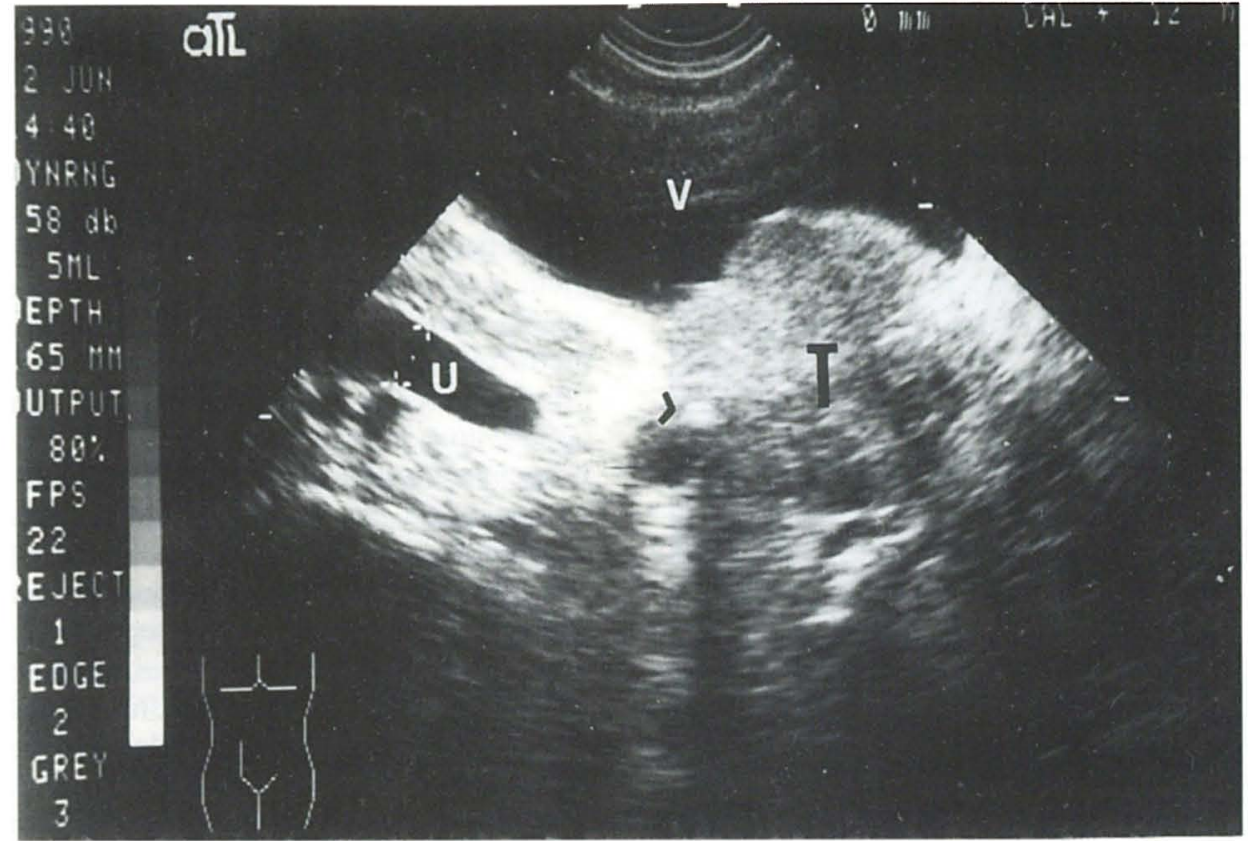

Fig. 3 - Scansione longitudinale anteriore dell' ipogastrio eseguita con paziente in decubito supino. Si rileva voluminosa formazione eteroplastica $(T)$ di pertinenza del corpo uterino, ad ecostruttura disomogenea, nel cui contesto è riconoscibile una piccola immagine iperecogena con cono d'ombra compatibile con calcificazione intraneoplastica (>). Il tumore sito in vicinanza del trigono vescicale per infiltrazione determina retrodilatazione ureterale $(U)$ bilaterale con idronefrosi settata a destra. $V=V e s c i c a$.

alterato funzionamento del rene controlaterale che, non più in grado di compensare la funzionalità renale, slatentizza un quadro di nefropatia bilaterale.

Il quadro iconografico è quello della idronefrosi severa, caratterizzato da dilatazione della pelvi e dei calici con ripercussione sul parenchima renale. Possiamo distinguere un aspetto settato in cui le grandi aree anecogene pieliche in contatto con il parenchima renale a spessore ridotto conservano ancora la loro individualità, ed un aspetto a sacca in cui i calici sono inglobati nella pelvi notevolmente ectasica fino a formare una unica e voluminosa area anecognea (3).

La calcolosi renale costituisce il 15-20\% delle affezioni urologiche, con una incidenza in Italia di 200 casi all'anno ogni 100.000 abitanti (9).

La natura chimica della litiasi non ha alcuna influenza sull'immagine ecografica, la cui visibilità dipende invece dalle dimensioni del calcolo, dallo spessore e dalla natura del tessuto interposto tra calcolo e trasduttore, e dalla posizione del calcolo rispetto al fuoco della sonda (10).

La litiasi renale è di norma rappresentata da un'immagine iperecogena più o meno omogenea, talora sotto forma di una placca, con ombra acustica posteriore. Tale ombra acustica dipende anch'essa da molti fattori quali l'incidenza del fascio di ultrasuoni sul calcolo, le dimensioni e la struttura cristallina del calcolo, la frequenza e la focalizzazione del trasduttore, e soltanto eccezionalmente può risultare non evidente come in piccole formazioni litiasiche di $2-3 \mathrm{~mm}$.

L'ecotomografia ha una sensibilità del $90-95 \%$ ed una specificità dell' $85-90 \%$ per calcoli superiori ai $3 \mathrm{~mm}$ (9).

Possiamo ecograficamente distinguere le nefrolitiasi, condizione in cui il calcolo è presente nel calice o nel bacinetto, dalla urolitiasi quando il calcolo è in qualsiasi altro punto della via escretrice (10).

Nella calcolosi caliciale o pielica semplice in assenza di idronefrosi o con concomitante ectasia pielocaliciale rileviamo l'immagine iperecognea in posizione declive all'interno di una formazione transonica, talora mobile con le variazioni posturali (litiasi secondaria).

Nelle litiasi coralliforme a stampo rileviamo invece una disorganizzazione dell'ecostruttura pielica.

La sintomatologia tipica della litiasi renale è l'episodio di colica che si manifesta con violento dolore lombare irradiato alla regione inguinale omolaterale, tene- smo vescicale, stranguria e pollachiuria, spesso ematuria micro o macroscopica, talora sintomi gastroenterici, e piressia per complicanze settiche. La mobilizzazione del calcolo lungo la via escretrice è solitamente causa di dolore tipo colico ed è spesso possibile documentare l'immagine iperecognea con il caratteristico cono d'ombra in fossa iliaca in sede paravescicale a livello del tratto terminale dell'uretere oppure a livello dell'ostio intramurale.

Un altro grande capitolo delle urgenze nefrologiche è rappresentato dalle complicanze delle lesioni cistiche. Le cisti renali sono lesioni cistiche vere, congenite o acquisite, presenti in quasi il 50\% dei soggetti al di sopra dei 50 anni (10, 11) ad eziologia ignota e solitamente asintomatiche.

Reperti del tutto occasionali di indagini ecotomografiche ed urografiche, talvolta le cisti diventano sintomatiche per complicanze quali l'infezione, l'emorragia, la rottura oppure l'ostruzione ureterale per compressione a causa delle notevoli dimensioni fino a diventare talvolta una massa palpabile responsabile di senso di peso, lombalgie ed ematuria.

Possono presentare sede corticale e sviluppo estrinseco polare o mesorenale, o sede intraparenchimale, oppure centrale o iuxtapieliche.

Generalmente all'ecotomografia si presentano come immagini anecogene, tondeggianti ovalari, a contorni netti e regolari, pareti sottili, contenuto transonico, spesso con un incremento della trasmissione del segnale o rinforzo della parete posteriore, e coni d'ombra laterali.

Alterazioni possono riguardare la forma (irregolarità di contorno), la parete (aumento di spessore) o il contenuto (diminuzione della transonicità).

L'indagine ecografica rileva una cisti complicata o infetta come un'immagine ipo-anecogena con un raggruppamento di echi grossolani più o meno omogenei e/o setti più o meno spessi in essa contenuti.

Nell'emorragia cistica mostra un contenuto inizialmente ecogeno e successivamente tale ecogenicità si riduce (lisi delle emazie) per aumentare nuovamente a distanza di tempo per l'organizzazione e la fibrosi conseguenti, con comparsa anche di eventuali calcificazioni.

Un'altra complicanza seppur rara delle lesioni cistiche è rappresentata dalla degenerazione neoplastica con l'evidenza 
di vegetazioni tumorali intracistiche (12).

L'indagine ecotomografica possiede una elevata sensibilità, superiore all'urografia (10), per la patologia tumorale, pari al 93-98\% per i tumori medio grandi ed al $74-82 \%$ per i tumori di dimensioni medio-piccole, ed elevata accuratezza diagnostica (12).

Il tumore di Grawitz rappresenta oltre il $90 \%$ delle neoplasie maligne renali dell'adulto $(4,5)$. Il sintomo più frequente nei casi sintomatici è l'ematuria totale (40\% dei casi) indicativa di una invasione del sistema corticale, tipica anche dei tumori delle vie escretrici, associandosi talora a colica renale per l'espulsione di coaguli. Nel $10 \%$ dei casi è presente dolore lombare aspecifico secondario alla distensione della capsula, ostruzione del giunto pielo-ureterale o interessamento delle strutture contingue; massa palpabile nel $3 \%$ dei casi, più frequentemente nel bambino, e talora una sintomatologia paraneoplastica, febbre, dimagrimento.

Il quadro iconografico mostra una tumefazione a sede periferica a sviluppo estrinseco, con profilo renale deformato, che a volte sposta il rene dalla propria sede, oppure una bozza aggettante nel seno pielico con deformazione del limite netto tra parenchima e seno pielico; tali immagini richiedono una diagnosi differenziale con pseudotumori come l'ipertrofia della colonna del Bertin, ipertrofia compensatoria o rene a dromedario.

L'ecogenicità in confronto al parenchima sano è variabile in senso iso, ipo o anecogeno-cistico, (seppur con una prevalenza della ipoecogenicità) rendendo spesso difficile non solo l'approfondimento diagnostico ma anche il semplice riconoscimento. Nelle neoplasie di grosse dimensioni immagini ipo-anecogene talora con rinforzo di parete posteriore sono riferibili a necrosi colliquativa o emorragia non recente, e se visibili alla periferia indicative di sanguinamento o colata neoplastica.

Utile la diagnosi differenziale con l'oncocitoma, neoplasia renale benigna a quadro ecografico aspecifico e similare al T. di Gravitz, più spesso isoecogena omogenea, con eventualmente una cicatrice centrale, anche se difficilmente evidenziabile ecotomograficamente.

Valutare sempre in caso di patologia tumorale l'eventuale interessamento della vena renale omolaterale e della vena cava che possono essere infiltrate o trom- bizzate.

Un breve cenno anche alla patologia vascolare, causa anch'essa di urgenze nefrologiche. Mentre per l'occlusione completa del ramo principale dell'arteria renale è più sensibile l'ecodoppler quale indagine diagnostica consentendo di evidenziare l'assenza di flusso ematico, per la trombosi venosa l'ecotomografia riveste ancora il ruolo di ausilio diagnostico. Il rene è aumentato di dimensioni rispetto al controlaterale, presenta una diminuita ecogenicità parenchimale ed aree di disuniformità ecostrutturali intraparenchimali, ed è visibile la vena occupata da materiale ecogeno endoluminale.

\section{L'ecotomografia nelle urgenze nefrologiche dell'anziano}

Nell'anziano, oltre alle affezioni nefrologiche comuni all'adulto, vi sono patologie più caratteristiche di tale periodo di vita quali l'ipertrofia prostatica, le neoplasie prostatiche ed uterine, le neoplasie vescicali oppure patologie neurologiche determinanti ostruzioni disfunzionali ed ancora la necrosi papillare tipica nei pazienti diabetici.

L'ipertrofia prostatica è un'affezione frequente in soggetti che hanno superato i 50 anni; colpisce il 50-60\% dei soggetti tra 40-59 anni ed oltre il $95 \%$ di coloro che hanno superato i 70 anni. La sintomatologia è la seguente: pollachiuria, nicturia, disuria, difficoltà ad iniziare la minzione ed ad arrestarla, perdita spontanea di urine da rigurgito, ed oligo-anuria in quei casi in cui la notevole ipertrofia comprime e stenosa il canale uretrale sì da causare l'ostruzione parziale o totale dell'uretra bulbare configurando un quadro di globo vescicale.

Le scansioni sovrapubiche, pur con una serie di limitazioni (sede dell'organo caudalmente nel bacino, in gran parte nascosto dalla sinfisi pubica), risultano di ausilio nel rilevare la presenza o meno di aggetti endovescicali della base prostatica consentendo la differenziazione dell'ipetrofia bilobata simmetrica dall'ipertrofia del terzo lobo (non valutabile con l'esplorazione rettale) e dall'ipertrofia trilobata. La prostata aumentata nelle sue dimensioni (diametro antero-posteriore, trasversale e longitudinale) mostra nelle sezioni trasverali un'area centrale in genere lievemente ipoecogena rotondeggiante riferibile all'adenoma, circondata posteriormente da una fascia parenchimale più ecogena riferibile alla zona periferica compressa dall'adenoma stesso. L'ostruzione cervico-prostatica inveterata determina una ipertrofia del muscolo detrusore vescicale ed un ispessimento irregolare dell'intera parete vescicale, con una tipica sintomatologia da vescica da sforzo e con ristagno postminzionale. La patologia neoplastica prostatica, al contrario dell'adenoma, presenta sede elettiva periferica nel $95 \%$ dei casi. L'ecotomografia presenta una sensibilità dell' $89 \%$, una specificità del $50 \%$ ed una certezza diagnostica del $63 \%$ (14). Iconograficamente si presenta come una immagine solida rotondeggiante-ovalare o a "placca", ipoecogena (a prevalente componente stromale) similmente all'adenoma, per cui sulla base della sola ecogenicità non è possibile distinguere un nodulo ipertrofico da uno neoplastico (15). Nelle fasi successive si ha l'interessamento della capsula ghiandolare, che viene infiltrata ed interrotta, con coinvolgimento dei piani periprostatici. Nelle neoplasie avanzate si rileva un completo sovvertimento dell'aspetto ecografico normale con irregolarità soprattutto del parenchima periferico. Attualmente grazie all'impiego delle sonde transrettali e con l'ausilio dei marker tumorali (PAP, PSA) sono sempre più rari quei quadri di oligo-anuria determinati da neoplasie di grosse dimensioni infiltranti il trigono vescicale ed il tratto intramurale ureterale, e la diagnosi è sempre più precoce con riconoscimento di forme neoplastiche di piccole dimensioni. Non lo stesso può dirsi per i tumori uterini, i quali presentano talora una sintomatologia più sfumata e meno significativa dal punto di vista urologico, concretizzando la possibilità, ancora ai giorni nostri, di visualizzare ecotomograficamente voluminose neoformazioni come 1'esempio che $\mathrm{Vi}$ offriamo nella Figura 3. Il tumore della vescica si presenta all'ecotomografia come un'area disomogenea per le aree di necrosi intratumorali, ecogena, a contorni sfrangiati nelle forme vegetanti oppure tozza ed a contorni più regolari nelle forme solide, senza segni di attenuazione posteriore ad eccezione di quando contiene depositi calcifici, fissa ed aderente alla parete vescicale. Neoplasie di grosse dimensioni possono determinare una ste- 
nosi parziale o serrata di un uretere determinando retrodilatazione ed idronefrosi, e sintomi urologici.

\section{BIBLIOGRAFIA}

1. Sperandeo G. Le nefropatie acute. In: Campani R, Gortenuti G, Micali M, Talia B, eds. Ecografia dell'apparato urogenitale. Torino: Minerva Medica 1992; 73-5.

2. Capaccioli L, Bartolini M, Lascialfari L eds. Patologia Pediatrica. In: Campani R, Gortenuti G, Micali M, Talia B, eds. Ecografia dell'apparato urogenitale. Torino: Minerva Medica, 1992; 147-60.

3. Perale R, Talenti E, Rubaltelli L, Candiani F. Ecotomografia renale in età pediatrica. In: Ziviello $M$, Biggi E, Ferrari F, Maresca G, Rubatelli L, eds. Ecoto-mografia. Napoli: Idelson. 1990; 316-43.

4. Robbins SL, Gotran RS, Kumar V. Le basi patologiche delle malattie. II Ed. Piccin 1987.

5. Bonomini V, Vangelista A, Stefoni S. Nefrologia Clinica. II Ed. 1993; 7: 155-79.

6. Glazer GM, Callen PW, Filly RA. Medullary nephrocalcinosis: sonographic evaluation. AJR 1982; 138: 35-7.

7. Brennan JN, Diwan RY, S.P. Makker et al. Ultrasonic diagnosis of primary hyperoxaluria in infancy. Radiology 1982; 145: 147-8.

8. Principi N, Edefonti A. Uropatie ostruttive e malformazioni, dell'apparato uropoietico. In: T. Schwarz ed. Manuale di Pediatria; 1988: IX Ed. 864-9.

9. Rosi P. La calcolosi renale. In: Campani R, Cortenuti G, Micali M, Talia B eds. L'ecografia dell'apparato urogenitale. Torino: Minerva Medica 1992; 81-6.
10. Bazzocchi M, Brizzi F, Cressa C, et al. Reni. In: Ziviello M, Biggi E, Ferrari F, Maresca G, Rubaltelli L eds. Ecotomografia. Napoli: Idelson 1990; 250-99.

11. Niselli A, Bacchini E. Le cisti renali. In: Campani R, Gortenuti $\mathrm{G}$, Micali M, Talia B eds. L'ecografia dell'apparato urogenitale. Torino: Minerva Medica 1992; 99-105.

12. Campani R, Bottinelli $\mathrm{O}$. Tumori renali: Problemi di diagnostica differenziale. In: Campani R, Gortenuti G, Micali M, Talia B eds. L'ecografia dell'apparato uro-genitale. Torino: Minerva Medica 1992; 119-25.

13. Rabassini A. Eco-color doppler: applicazioni in campo uronefrologico. In: Campani R, Gortenuti G, Micali M, Talia B eds. L'ecotomografia dell'apparato urogenitale. Torino: Minerva Medica 1992; 67-72.

14. D'Eramo G. Carcinoma prostatico. In: Campani R, Gortenuti G, Micali M, Talia B eds. L'ecotomografia dell'apparato urogenitale. Torino: Minerva Medica 1992; 215-9.

15. Gualdi G, Burrai L, Conforti G, et al. Vescica e prostata. In: Ziviello M, Biggi E, Ferrari F, Maresca G, Rubaltelli L eds. Ecotomografia. Napoli: Idelson 1990; 344-71. 